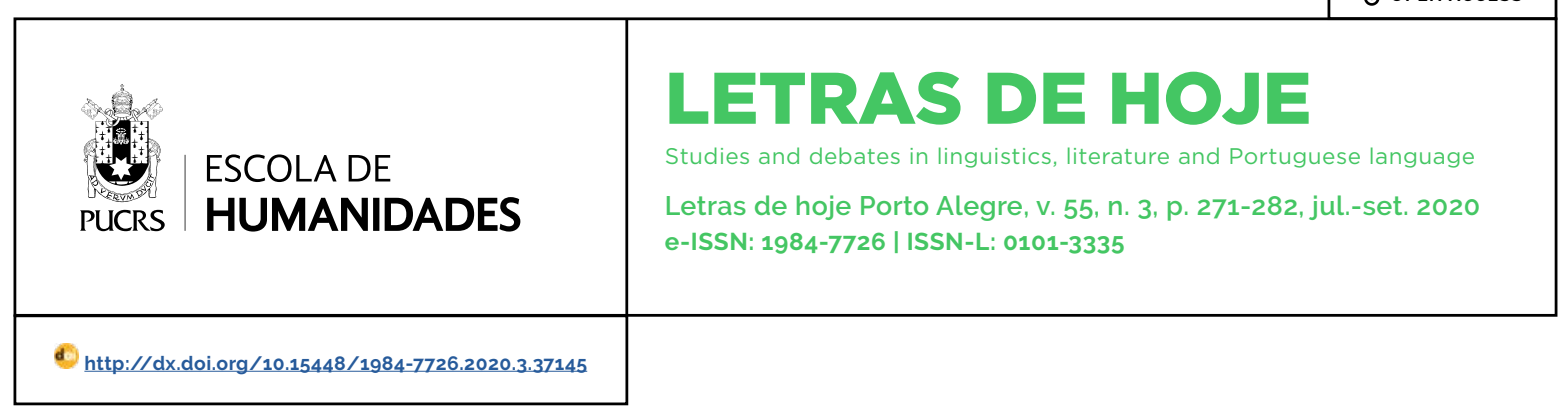

TEMÁTICA LIVRE

\title{
Representaciones de las lenguas en La terquedad de Rafael Spregelburd
}

\author{
Representações das linguas na La terquedad de Rafael Spregelburd \\ Languages representations in La terquedad by Rafael Spregelburd
}

\author{
Mariano Nicolás Zucchi ${ }^{1}$ \\ orcid.org/0000-0003-4245-2585 \\ marianonzucchi@gmail.com
}

Recebido em: 24/02/2020 Aprovado em: $11 / 08 / 2020$ Publicado em: 17/12/2020
Resumen: La terquedad (SPREGELBURD, 2009) es un texto dramático en el que conviven diferentes lenguas. Este artículo, que se inserta en una investigación de doctorado en curso, pretende evaluar qué representaciones emergen en la pieza de los diferentes sistemas lingüisticos que se ponen en escena. Concretamente, en primer lugar, buscaremos probar que, mientras que el inglés emerge como una lengua internacional, el valenciano aparece representado, en una situación de diglosia, como poco prestigioso. En segundo lugar, intentaremos mostrar que las diferentes variedades del español de las que se vale el material se presentan como pertenecientes a un sistema relativamente homogéneo en consonancia con la tradición discursiva panhispanista. Finalmente, en cuanto a la perspectiva teórica, nos enmarcaremos en el Enfoque dialógico de la argumentación y la polifonia (GARCÍA NEGRONI, 2009; 2016a; 2016b; 2018; 2019), teoria no veritativista de la significación y no unicista del sujeto.

Palabras clave: Spregelburd. representaciones lingüisticas. español. panhispanismo. Real Academia Española.

Resumo: La terquedad (SPREGELBURD, 2009) é um texto dramático em que diferentes idiomas coexistem. Este artigo, inserido em uma pesquisa de doutorado em andamento, tem como objetivo avaliar quais representações emergem na peça dos diferentes sistemas linguísticos encenados. Especificamente, em primeiro lugar, procuraremos provar que, enquanto o inglês emerge como idioma internacional, o valenciano é representado, em uma situação de diglossia, como irreconhecivel. Segundo, tentaremos mostrar que as diferentes variedades de espanhol que o material utiliza são apresentadas como pertencentes a um sistema relativamente homogêneo, alinhado à tradição do discurso pan-hispânico. Por fim, em relação à perspectiva teórica, seremos enquadrados na Enfoque dialógico de la argumentación y la polifonia (GARCÍA NEGRONI, 2009. 2016a; 2016b; 2018; 2019), teoria não veritativista do significado e não unicista do sujeito. Palavras-chave: Spregelburd. Representações Linguísticas. Espanhol. Panispanismo. Real Academia Española.

Abstract: La terquedad (SPREGELBURD, 2009) is a dramatic text in which different languages coexist. This article, which is inserted in an ongoing doctoral research, aims to evaluate which representations of each linguistic system emerge from this play. First, we will try to show that English appears as an international language, while Valencian is represented as non-prestigious dialect in a diglossia situation. Second, we will intend to probe that the different varieties of Spanish used in the material are presented as belonging to a relatively homogeneous system, showing that the text is in line with the Pan-Hispanic discourse tradition. Finally, regarding the theoretical perspective, we will be framed in the Enfoque dialógico de la argumentación y la polifonia (GARCIA NEGRONI, 2009; 2016a; 2016b; 2018; 2019), a non-veritativist theory of the linguistic meaning.

Keywords: Spregelburd. Linguistic Representations. Spanish. Panhispanism. Royal Spanish Academy. 


\section{Introducción}

Este trabajo se enmarca en una investigación de doctorado en curso que pretende relevar el mecanismo enunciativo mediante el cual la subjetividad autoral queda plasmada en el género texto dramático. Especificamente, y enmarcados en una perspectiva dialógica y polifónica de la enunciación (El Enfoque dialógico de la argumentación y la polifonía, en adelante, EDAP (GARCÍA NEGRONI, 2009; 2016a; 2016b; 2018; 2019)), nuestra indagación toma como corpus de trabajo la Heptalogía de Hieronymus Bosch del dramaturgo argentino Rafael Spregelburd y busca examinar la manera en que los siete textos dramáticos construyen una imagen de su autor en tanto responsable de la enunciación global.

Al respecto, el análisis preliminar del material reveló que las últimas obras de la serie (El pánico, La estupidez, La paranoia y La terquedad) ponen en escena una representación del dramaturgo emparentada con la figura de un ethos intelectual (cf. ZUCCHI, 2018 y 2019b). En particular, en La terquedad (2009) $)^{2}$, texto que constituye el corpus de este trabajo, el responsable de la enunciación global emerge como un sujeto multilingüe. De hecho, en Zucchi (2019a) mostramos que la gran cantidad de sistemas lingüisticos que se utilizan en la pieza (tanto en las réplicas de los personajes como en las didascalias y notas al pie) se orienta a generar una representación del dramaturgo como la de alguien con un alto conocimiento en lenguas. Es más, el saber que se le atribuye a esta persona del discurso cumple la función de legitimar su voz y el posicionamiento burlesco con el que queda presentado frente a los personajes de la diégesis.

En ese sentido, este artículo se propone como una profundización de dicha línea de investigación, especificamente, mediante el estudio de las representaciones asociadas a cada una de las variedades que se ponen en escena en la obra. Desde nuestra perspectiva, identificar estos valores atribuidos a las lenguas, con los que el dramaturgo (como persona del discurso) queda homologado, es fundamental al momento de describir las particularidades de su figura. Asi pues, nuestra indagación pretende, en primer término, relevar qué sistemas lingüísticos aparecen en la obra y qué función se le otorga a cada uno de ellos. Para ello, nos centraremos en el estudio de las notas al pie, enunciados que nos permitirán determinar si las lenguas empleadas se muestran como conocidas por parte del lector modelo del texto ${ }^{3}$. En segundo término, examinaremos las distintas variedades del español utilizadas en la pieza, buscando identificar cuál de ellas se le atribuye al responsable de la enunciación global y qué lugar y valor se les asigna a las demás.

A modo de hipótesis general, pretendemos probar que, de las tres lenguas empleadas mayoritariamente en el texto (el inglés, el valenciano y el español), la primera queda representada como una lengua conocida por el lector modelo del texto y asociada a la función de lengua internacional. En cuanto a la segunda, el modo específico en que es presentada, como el sistema lingüistico de menor estatus en una situación de diglosia español-valenciano ${ }^{4}$, permite caracterizarla como una lengua poco prestigiosa. En otras palabras, se observará que este sistema emerge como con un alcance estrictamente regional y, en ese sentido, como de menor importancia en relación a las demás variedades que se utilizan en el texto. Finalmente, y a propósito del español, estudiaremos, en primer lugar, cómo el texto dramático escenifica tres variantes diferentes de esta lengua (el español

\footnotetext{
2 Para el presente artículo decidimos trabajar con la edición de 2009 de Atuel del texto dramático. Todas las citas serán extraídas de ese volumen y se indicará a continuación de cada una de ellas y entre paréntesis el número de página. En todos los casos, las negritas son nuestras.

3 Según Eco (1981) todo texto de ficción construye una imagen de su destinatario ideal. Para dar cuenta de esta dimensión el teórico italiano propone el concepto de lector modelo y lo define como un conjunto de condiciones de felicidad establecidas textualmente que deben satisfacerse para que un texto quede plenamente actualizado. En otras palabras, el lector modelo, entidad de naturaleza virtual, resulta como el individuo capaz de decodificar aquellos contenidos y procedimientos que la obra pone en escena.

4 Según Ferguson (1959), la diglosia es una situación lingüística conflictiva caracterizada por la coexistencia de dos lenguas en una comunidad hablante. Cada uno de estos sistemas lingüisticos, por su parte, posee usos y valores asociados diferentes. En términos generales, una de las variedades es considerada más prestigiosa, es empleada para comunicaciones oficiales y administrativas y enseñada en el sistema escolar. La otra, restringida al universo de los intercambios familiares, suele ser considerada con un estatus inferior y, con el tiempo, tiende a no ser aprendida por las nuevas generaciones.
} 
peninsular, un supuesto "español general" y una variedad americana presuntamente rioplatense). Al respecto, nos interesará sugerir que la convivencia en apariencia "armoniosa" de estos sistemas en la pieza - y la propia puesta en escena de un español de tipo "general"- permiten leer que la obra se homologa con la tradición discursiva del panhispanismo. En segundo lugar, el análisis del lenguaje utilizado en el texto primero nos permitirá mostrar que en La terquedad el "español general" no queda mostrado como un sistema homogéneo. En efecto, la alternancia en algunos rasgos presentes en las réplicas (en particular, en el uso de los pronombres átonos de tercera persona en posición objeto directo y de la segunda persona del singular y del plural en modo imperativo) evidencia que esta variedad no queda representada como una lengua estable. A propósito de este punto, nos interesará sugerir que la vacilación en torno a las características que definen a esta "lengua común" puede explicarse como una consecuencia directa de la sintonía que la obra parece expresar con el ideal panhispánico.

Para probar nuestra hipótesis, en primera instancia debemos definir el marco teóricometodológico desde el cual analizamos nuestro objeto de estudio. Como anticipamos, en este trabajo adoptaremos la perspectiva del EDAP, teoría no referencialista de la significación y no unicista del sujeto. Este enfoque pretende, esencialmente, describir el sentido de los enunciados en clave dialógico-argumentativa y, para ello, recupera (y reformula) alguna de las ideas fundantes de las teorias de corte enunciativo (La Teoria de la Polifonía (DUCROT, 1984), La Teoría de la argumentación en la lengua (ANSCOMBRE Y DUCROT, 1983), La Teoría de los bloques semánticos (CAREL Y DUCROT, 2005), La Teoría de las heterogeneidades enunciativas (AUTHIER, 1983) y el dialogismo bajtiniano (BAJTíN, 1985)).

A los efectos de la realización de este trabajo, nos interesa destacar que esta perspectiva rechaza considerar la intención y las particularidades psicofísicas del sujeto hablante como elementos determinantes del valor semántico-pragmático de una expresión (GARCÍA NEGRONI, LIBENSON
Y MONTERO, 2013). En efecto, para el EDAP estas no quedan reflejadas de forma transparente en el enunciado y, así, no deberían formar parte de la descripción de su sentido. En otras palabras, esta perspectiva teórica supone que la relación entre el hablante y el lenguaje es completamente opaca y, así, se distancia de aquellos enfoques que conciben el discurso como un espacio en el que pueden identificarse las huellas de sus condiciones materiales de producción (como hace el Análisis del discurso (PÊCHEUX, 2016), por ejemplo). En su lugar, esta perspectiva entiende a la subjetividad, básicamente, como un efecto de sentido que surge como consecuencia de la plasmación en el enunciado de una serie de perspectivas enunciativas (llamadas puntos de vista) y de discursos ajenos. Frente a estos, el Locutor (el responsable de la enunciación según el enunciado) quedará adoptando distintos posicionamientos (homologación, concesión, rechazo, entre otros).

En este marco, nos resulta imposible aceptar el concepto de representación, como lo hace el marxismo, en términos de una suerte de pantalla ideológica que media entre el sujeto y el objeto. En la misma línea, tampoco parece viable retomar para este trabajo la definición que da del concepto la sociolingüística tradicional (cf. MOSCOVICl, 1979; RAITER, 2001), perspectiva para la cual la representación se define en como: "el resultado de un acto cognitivo por medio del cual se produce un signo o símbolo que se instaura como el "doble" de una presunta "realidad" o de un "original"" (SZURMUK Y MCKEE IRWIN, 2009, p. 250). De naturaleza estrictamente mental, las representaciones constituirian para este enfoque un conjunto estable de ideas que tiene el hablante sobre los objetos del mundo, imágenes que según la sociolingüística y el análisis del discurso podrian rastrearse a partir del estudio de las producciones lingüisticas, lugar en el que quedarian inscriptas. Como puede anticiparse, si bien por supuesto el EDAP admite la existencia de representaciones de tipo mental, en términos metodológicos, no acepta que éstas puedan ser identificadas directamente en la materialidad verbal. En ese sentido, en este trabajo, aunque conservaremos 
el concepto de representación, lo entenderemos como una noción de dominio estrictamente discursivo, definida por el modo particular en que un determinado objeto (o situación) queda caracterizada según un enunciado 5 .

Ahora bien, dejando de lado estas precisiones metodológicas, en este artículo nos centraremos estrictamente en el análisis de las representaciones sobre las lenguas, constructos que, como desarrollaron extensamente los trabajos en el marco de la glotopolítica (cf., entre otros, ARNOUX, 2010; FASOLD, 1996; FISHMAN, 1970; GALLARDO, 1978), tienen un rol fundamental en la implementación de políticas lingüisticas y procesos de estandarización. Al respecto, Woolard (2007) propone que las ideas sobre las lenguas se nuclean en dos "ideologías lingüísticas": la de la autenticidad (que ubica el valor de una variedad asociada a una comunidad especifica y como expresión de su identidad) y la del anonimato (que reproduce una representación de la lengua como un sistema homogéneo que desborda las fronteras de los estados nacionales y permite a hablantes de comunidades distantes comunicarse entre sí) ${ }^{6}$.

En el caso del español, la representación de la lengua ligada al valor del "anonimato" parece ser aquella presente en los discursos que formula la Real Academia Española (en adelante, RAE) como parte de su política lingüística. En efecto, desde la década de 1990 los instrumentos de la Académica se orientaron a instaurar la idea de que existe una suerte de "español general", compartido por toda la comunidad hispanohablante: "El español, por su carácter de lengua supranacional, constituye en realidad un conjunto de normas diversas que, no obstante, comparten una amplia base común" (NPLP, p. 9). Para reforzar este imaginario de lengua compartida, la RAE ha empleado una serie de ideologemas que fueron los ejes de los congresos de la lengua española: "la lengua es la patria", "nuestra lengua es mestiza" y "el español es americano". Analizados exhaustivamente por Arnoux (2006), estos lemas muestran el esfuerzo de la Academia en construir una representación de la lengua y de la comunidad hispanoamericana como una unidad más allá de sus diferencias:

\begin{abstract}
Es por ello la expresión culta formal la que constituye el español estándar: la lengua que todos empleamos, o aspiramos a emplear. cuando sentimos la necesidad de expresarnos con corrección [...] Es, en definitiva, la que configura la norma, el código compartido que hace posible que hispanohablantes de muy distintas procedencias se entiendan sin dificultad". (DPD, p. XVI)
\end{abstract}

Ahora bien, si existe una lengua común, dicho sistema también estará regulado por una única norman (llamada Norma panhispánica) y por una única institución, la RAE. Por supuesto que la construcción de una representación de la lengua en este sentido no parece ser inocente: es una forma de, por un lado, mantener el foco normativo en Madrid y, por otro, controlar el mercado de la lengua. Dice Lauria (2018, pp. 178-179):

El modelo panhispánico académico actual
declara ampliar la base del estándar y avanza,
con reservas, en la aceptación de variantes
regionales [.... Si bien acusa la construcción
de un estándar supranacional, numerosos
estudios críticos develan, a partir del análisis
de discursos metalingüisticos, su anclaje ideo-
lógico en cuanto a la gestión pretendidamente
"colectiva y democrática" de la lengua, la cual,
en realidad, perdura bajo la tutela centralista
de Madrid y al tratamiento otorgado a las va-
riedades, en especial, a "lo americano" en un
lugar subsidiario.

En este marco, como muestran Lauria y López García (2009), los instrumentos creados por la RAE no hacen otra cosa que presentar como "propios del español" aquellos rasgos típicamente castellanos, mientras que las particularidades lingüísticas de las variedades americanas quedan subsumidas a la categoria de variantes regionales.

\footnotetext{
5 Si bien queda por fuera de los limites de este trabajo, debemos decir que desde nuestra perspectiva esta "caracterización" es esencialmente argumentativa y puede realizarse en términos de un encadenamiento argumentativo, esto es, un binomio que se construye siempre a partir de dos segmentos unidos por un conector. Este último puede ser de dos tipos: "normativo" (por ejemplo, "por lo tanto") o "transgresivo" (por ejemplo, "sin embargo") (CAREL Y DUCROT, 2005).

6 Si bien para la perspectiva que adoptamos aqui es problemático utilizar el concepto de "ideologia", nos resulta interesante recuperar la perspectiva de Woolard y pensarla en términos de tradiciones discursivas evocadas por los enunciados al momento de construir representaciones sobre las variedades.
} 
Así, bajo un discurso que dice valorar la diversidad lingüistica del español y poner en pie de igualdad todos los dialectos que forman parte del sistema, el panhispanismo reproduce los antiguos ideales de pureza de la lengua.

Sin embargo, y aquí radica el punto que nos interesa destacar, el "español general" es, en sentido estricto, un constructo teórico, un sistema lingüístico que no es empleado por los hablantes. De hecho, se funda en una representación ideal de la comunidad lingüistica hispana, que queda presentada, esencialmente, como homogénea. En consecuencia, el hecho de que los instrumentos normativos estén orientados a describir una variedad que no es utilizada efectivamente en los intercambios comunicativos produce un vacio descriptivo. En efecto, las distintas variedades del español no reciben, al menos por parte del discurso "oficial", la atención necesaria y esto se traduce en la ausencia de caracterizaciones sistemáticas de la forma y el funcionamiento de dichos sistemas lingüísticos. En otros términos, la reproducción de la norma panhispánica no solo genera desconocimiento de las particularidades de cada una de las variedades del español, sino que, además, dificulta la identificación de las diferencias entre los distintos dialectos de la misma lengua?.

Como intentaremos probar, este mismo escenario se replica en La terquedad. En efecto, la libre alternancia entre distintas variedades del español y la puesta en escena de un "español general", que se presenta como fácilmente reconocible, parecen reproducir el ideal panhispánico. Sin embargo, este sistema, en tanto lengua artificial creada en la obra, presenta alternancias entre rasgos de distintos dialectos que evidencian que se trata de una variedad inestable. Desde nuestra perspectiva, este hecho refuerza la homologación del texto con el discurso panhispánico, el cual, enfocado en la descripción de una "base común", no concibe las particularidades de cada variedad como un elemento que atente contra la comunicación entre los distintos miembros de la comunidad hispanohablante.

\section{Representaciones de las lenguas en La terquedad}

Como se anticipó, La terquedad es un texto dramático que constituye una matriz esencialmente multilingüe. En efecto, conforman su textualidad un conjunto numeroso de variedades que se van alternando en función de quiénes sean los personajes que intervienen en la acción dramática. Esta diversidad hace, de algún modo, que una reflexión en torno a los valores asociados a las lenguas sea pertinente al momento de intentar analizar la construcción de sentido en el material. Sin embargo, la pluralidad de variedades presentes no es el único elemento del texto que justifica una indagación como la que nos proponemos. De hecho, el núcleo conflictual de la pieza también se concentra en un problema de orden lingüístico: en la ciudad de Valencia, en 1939 y en el marco de la Guerra Civil Española, Planc, el comisario del pueblo, dice haber descubierto una lengua innata, cuyo empleo garantizaría una comunicación exitosa por parte de todos los miembros de la especie. En un intento de estandarizar este "hallazgo", junto con su hija desarrolla un diccionario, el cual motiva la visita del ruso Dmitri. Con su llegada emergen, en los demás personajes, sospechas de vínculos con el régimen comunista.

Tal como dijimos en nuestra introducción, en lo que sigue analizaremos qué representación emerge del inglés, del valenciano y de las distintas variedades del español, los tres sistemas lingüísticos usados mayoritariamente en el texto ${ }^{8}$.

En cuanto al inglés, el Acto II es el que presenta

\footnotetext{
7 López García (2015) analiza este fenómeno en el marco del español rioplatense, en el que la acción de las Academias crea, según la autora, una suerte de "hiato normativo". Así, el desprestigio asociado históricamente al español americano y la ausencia de una gramática que describa exhaustivamente la variedad local genera un sentimiento de inseguridad lingüistica que lleva a los hablantes a recurrir al modelo peninsular como parámetro de corrección.

8 Debemos aclarar, sin embargo, que estas no son las únicas lenguas que aparecen en la obra. En efecto, Natalie, la mucama de la propiedad en la que transcurre la acción, habla en algunas ocasiones en francés. Además, Planc pronuncia un enunciado en Tupal (lenguaje artificial que es presentado bajo el rótulo de "Katak") (p. 43) y, al momento de explicar su "descubrimiento", traduce la palabra "padre" a once lenguas europeas (pp. 84-85).
} 
un número importante de enunciados en esta variedad. En estas escenas, se muestra el encuentro entre Fermina, la hija de Planc, y John Parson, un inglés que llega a la propiedad en búsqueda de una lista de presuntos simpatizantes del comunismo que serán ejecutados por el ejército español. Estos personajes se comunican esencialmente en inglés, alternándolo con el español en los momentos en que interviene el Padre Francisco, otros de los protagonistas. Lo que nos interesa destacar es que todos los enunciados que aparecen en esta lengua no poseen una nota al pie en la que se traduzca su significado al español. En consecuencia, la obra muestra a esta variedad como completamente comprensible para el lector modelo del texto. Este, por su parte, aparece representado entonces como un sujeto bilingüe español-inglés. En realidad, en la medida en que la mayor parte de la obra está escrita en español, podemos hipotetizar que el lector modelo es presentado, en verdad, como hablante nativo de español y que posee el inglés como lengua extranjera. En efecto, la complejidad lingüística que surge como resultado de la yuxtaposición de distintas variedades del español constituye un argumento para sostener que el lector modelo queda construido como con una gran competencia en el manejo de la lengua española. De cualquier modo, lo que nos interesa destacar aquí es que el modo en que el inglés se presenta como una lengua que no necesita traducción hace emerger una representación de este sistema como lengua internacional, esto es, como uno conocida por buena parte de la población mundial y, en tanto tal, como garante de intercambios comunicativos exitosos por parte de hablantes de lenguas diferentes. Como se sabe, esta representación del inglés es la que circula en buena parte de los discursos sociales ${ }^{9}$. Al respecto, nos interesa simplemente hacer notar que el uso especifico que se hace de esta variedad en la obra no solo recupera esta tradición discursiva, sino que la reproduce.

En cuanto al valenciano, este sistema lingüístico es utilizado sobre todo en el Acto III. Son los personajes de Primitivo 1 (Sanchis) y Primitivo 2 (Antoni) (nativos valencianos disfrazados de hombres prehistóricos) los que se comunican principalmente en esta lengua. También lo hacen Nuria y Antoni, quienes poseen un vínculo amoroso secreto. Ellos utilizan el valenciano solamente cuando se encuentran en soledad. Finalmente, el protagonista de la pieza, Planc, también profiere un enunciado en esta lengua en presencia de Dmitri. En todos los casos, la obra presenta una nota al pie, que queda a cargo del responsable de la enunciación global, en la que se provee una traducción de los dichos de los personajes al español. Así, y a diferencia de lo analizado en el párrafo anterior, el hecho de que la obra incorpore estas glosas aclaratorias configura al lector modelo como alguien que no conoce esta lengua y, al mismo tiempo, al valenciano como un sistema lingüistico limitado a una comunidad hablante restringida. Al respecto, y como anticipamos en nuestra introducción, el modo específico en que se muestra la alternancia español/valenciano parece reproducir una situación típica de diglosia. Nótese, a propósito de este punto, que la acción dramática transcurre en territorio de Valencia y, en ese sentido, sería esperable que los personajes, nativos de esa región, se comuniquen entre ellos en esa lengua. Sin embargo, si bien la obra los presenta como conocedores de ese sistema lingüistico (al menos, a Sanchis, Antoni, Nuria y Planc) es el español el elegido para la mayoría de sus intercambios. Esto, por su parte, hace emerger una representación del valenciano como una lengua de poco prestigio, ya que queda restringida a intercambios limitados y de extrema cotidianeidad. El español, en cambio, emerge como una lengua de mayor estatus, ya que es la utilizada, por ejemplo, para comunicarse con el ruso Dmitri que, por su conocimiento

\footnotetext{
9 Para explicar la pregnancia de esta representación del inglés, Bein introduce el concepto de fetiche lingüistico. Esta noción alude al proceso mediante el cual "a las lenguas se les atribuyen ciertas cualidades esenciales que son, en realidad, un reflejo de las funciones que desempeñan en ciertas relaciones sociales de producción" (2005, p. 5).
} 
rudimentario de esta variedad, parece haberla adquirido como lengua segunda ${ }^{10}$.

Sin embargo, las notas al pie de página aportan más información que, a modo de clave interpretativa, permite evaluar qué representación emerge de las variedades en la obra, en especial, del español. Consideremos los siguientes ejemplos:

(1) 17. "Estáis espléndidos, los dos, con esas pieles tan monas". (p. 95)

(2) 20. “Sigue muy mala?". (p. 95)

(3) 23. "Oh, no. Esto es sólo el comienzo. Luego vendrán las conferencias, y los libros, y todo eso, conozco muy bien todo ese rollo, Antoni". (p. 95)

Como puede constatarse, las tres muestras reproducen en la variedad peninsular del español lo que los personajes de la diégesis comunicaron en valenciano En efecto, el uso específico de la segunda persona del plural (conjugada con la persona "vosotros") y la ocurrencia de las expresiones "tan monas", "muy mala" y "ese rollo", típicamente castellanas, funcionan como evidencia que muestra que la variedad utilizada para la traducción es la peninsular. Ahora bien, ¿qué efecto de sentido genera esta operación? En principio, el hecho de que esta sea la lengua de destino en el proceso de traducción configura al lector modelo como alguien competente en esta variedad ${ }^{1112}$.

Con todo, para comprender qué representación emerge del español en la pieza debemos primero analizar qué variedades se utilizan en los otros dos tipos de enunciados que tradicionalmente aparecen en un texto dramático. En lo que sigue, entonces, nos concentraremos en estudiar las particularidades enunciativas de las didascalias y las réplicas.

En cuanto a las primeras, en principio, debemos decir que están escritas en lengua española. Sin embargo, y seguramente por las propias características lingüísticas de este tipo de enunciados (que, recordemos, tienden a estar escritos en tercera persona del singular del tiempo presente del modo indicativo y evitan la aparición de subjetivemas) ${ }^{13}$, es dificultoso identificar qué variedad se utiliza en ellos. Al respecto, es importante mencionar que, según la bibliografía especializada (cf. LAPESA, 1984), las diferencias más nítidas entre los distintos dialectos del español se encuentran en el nivel fonológico (žeísmo, seseo, yeísmo, aspiración, debilitamiento o caída de la /s/, comportamiento general de las palatales, curvas de entonación, entre otros), en las formas de tratamiento, en los tiempos verbales y en el léxico. De todos estos elementos, los primeros tres quedan neutralizados en el texto segundo como criterios de clasificación, ya que el uso extendido de la tercera persona del singular y del tiempo presente impiden registrar la posible variación. Sin embargo, es el léxico en este caso el que ayuda a resolver este problema:

(4) ([Planc] Descorre el velo que cubre al diccionario, y por primera vez vemos el armatoste. Bah, es una computadora. Un elemento totalmente anacrónico, muy parecido a nuestras computadoras actuales [...]). (p. 84)

Como puede observarse, los procedimientos lingüísticos presentes en (4) permiten identificar que la variedad utilizada en las didascalias es un

\footnotetext{
10 Este personaje incurre, de forma recurrente, en una serie de errores en la construcción de sus enunciados en español. En cada caso la obra incorpora una nota al pie con la sigla "sic" que pretende indicar que esas estructuras agramaticales deben atribuirse al personaje y no al responsable de la enunciación global. Esto, por su parte, y como analizamos en Zucchi (2019a), colabora en la construcción de una representación del dramaturgo como un intelectual, ya que queda mostrado como alguien con un conocimiento técnico sobre el lenguaje y homologado con un "modo de decir" correcto.

${ }_{11}$ En cuanto al uso de las comillas, que Authier (1984) interpreta como indices de heterogeneidad marcada, debemos decir que, en este empleo específico señalan que los enunciados no deben atribuirse al dramaturgo (que, en tanto responsable de la enunciación global. queda como a cargo de las notas al pie), sino a los personajes. En ese sentido, las comillas solo estarian indicando un posicionamiento de distancia respecto a los discursos, pero no a la variedad en la que ellos aparecen. De hecho, la elección del español peninsular como lengua de traducción genera por defecto una imagen del dramaturgo como la de alguien que conoce este sistema lingüístico. A propósito de este punto, el resto de las notas al pie que aparecen en el texto no poseen marcas lingüisticas que nos permitan identificar con nitidez con qué variedad del español queda homologado el responsable de la enunciación global en ellas.

12 En el marco de una comunicación personal, Spregelburd nos informó que esos enunciados aparecen en español peninsular ya que la persona encargada de realizar la traducción posee esa variedad como lengua materna. De cualquier modo, y en la medida en que adscribimos a una perspectiva no intencionalista, en este trabajo buscamos analizar el efecto de sentido que genera la ocurrencia de esta variedad más allá de las motivaciones efectivas que dieron lugar a su aparición.

13 Para una caracterización de las particularidades lingüísticas y enunciativas del texto segundo ver Zucchi (2019c).
} 
español americano (presuntamente rioplatense). En efecto, el lexema "computadora" presente en este enunciado muestra que las didascalias no están escritas en español peninsular (para el cual la opción no marcada es la palabra "ordenador")14. En relación a este punto, no es un dato menor que el lema en cuestión se presente en un enunciado en el aparezcan también la onomatopeya "Bah" (de marcado registro coloquial y, en tanto tal, ligada a un modo de decir típicamente vernáculo) y la primera persona del plural combinada con una marca deíctica ("nuestras computadoras actuales"). Estos procedimientos, en tanto marcas de subjetividad, ponen en primer plano la presencia de la figura del Locutor y evidencian su posicionamiento enunciativo. Justamente, la combinación de todos estos recursos nos permite inferir que el dramaturgo, que, recordemos, queda a cargo del discurso didascálico, es presentado por la obra como alguien que se expresa en una variedad americana del español. Al respecto, en la medida en que este sistema lingüístico tampoco es traducido, podemos afirmar que el lector modelo queda mostrado como alguien que también conoce esta variedad (junto con el inglés y el español peninsular, como analizamos más arriba).

Ahora bien, ¿qué sucede en las réplicas? ¿En qué lengua se desarrollan? Como anticipamos, el texto primero parece estar escrito en una suerte de "español general", esto es, uno que no posee marcas específicas que permitan asociarlo a alguna de las variedades vigentes en el territorio hispanohablante. Para ello, la obra se vale, básicamente, de aquellos rasgos que la mayoría de los dialectos tienen en común. Por ejemplo, las formas de tratamiento de segunda persona singular se ajustan a la persona gramatical "usted", opción presente en el conjunto de las variedades hispanoamericanas (aunque con distintos valores asociados dependiendo de cada caso). Algo similar ocurre con el sistema temporal del pasado. La variante más elegida para expresar este valor es la conjugación en pretérito perfecto compuesto, también común en muchas de las variedades del español ${ }^{15}$. En cuanto al léxico, el texto primero evita de forma sistemática el empleo de partículas asociadas específicamente a algunas de los dialectos. Con todo, el uso del pronombre "usted" (y la consecuente conjugación verbal en esa persona) en contextos de familiaridad y algunos casos en los que se conjuga la segunda persona del plural en "vosotros", hacen, al menos para el lector rioplatense, que el texto primero, sin llegar a estar escrito en la variedad castellana, suene "vagamente peninsular"16.

En relación a este punto, nos interesa realizar dos observaciones. En primer lugar, que este "español general" no aparece acompañado en ningún momento por algún tipo de nota al pie aclaratoria que explique sus características especificas. En ese sentido, podemos inferir que el lector modelo del texto queda presentado como competente en esta variedad (que, recordemos, en sentido estricto no es lengua materna de ningún individuo). Nótese, al respecto, la sincronía con el discurso panhispánico, para el que existe una suerte de "base idiomática común" que permite que todos los hispanohablantes puedan comunicarse entre sí. En segundo lugar, y en estricta relación con lo anterior, el hecho de que en el texto dramático se alternen tres variedades distintas del español (recordemos, el peninsular en las notas al pie, un dialecto americano en las didascalias y un "español general" en las réplicas) y que esto, en sí mismo, no sea presentado como conflictivo, muestra cierta homologación de la obra con el discurso de la

\footnotetext{
14 En cuanto a este punto, un estudio informal de la ocurrencia del lema nos permitió identificar que la palabra "computadora" se usa tanto en la variedad rioplatense como en la uruguaya y panameña. En ese sentido, no podemos afirmar con contundencia que el dialecto utilizado en las didascalias sea el español rioplatense (lengua materna del dramaturgo).

15 Al respecto, si bien no es el tiempo verbal más utilizado en la variedad rioplatense para expresar pasado, su uso se mantiene en los contextos en los que se expresa mayor grado de compromiso con lo expresado por la proposición. Un estudio detallado de los contextos de uso y los valores asociados al pretérito perfecto compuesto y su alternancia con el pretérito perfecto simple puede encontrarse en García Negroni (2009).

16 El propio Spregelburd, en la comunicación personal a la que nos remitimos más arriba, nos comunicó que su intención era justamente esa: no replicar la variedad peninsular (ya que no sabia a ciencia cierta qué características específicas tenía en 1939), sino poner en escena un sistema que remita a lo castellano para enfatizar el hecho de que la acción transcurre en tierra valenciana.
} 
RAE. En efecto, uno de los pilares de la Nueva política panhispánica (2004) es que, más allá de sus diferencias, todas las variedades del español comparten un número importante de rasgos que hacen que los hablantes de cualquiera de ellas comprendan perfectamente las demás. Como venimos argumentando, esto mismo sucede en La terquedad: la alternancia entre los tres sistemas y la ausencia de notas aclaratorias a propósito de los usos especificos de estos hacen emerger una imagen del lector modelo como la de alguien que comprende sin problemas las tres variedades del español. Si bien no podemos afirmar que esto muestra la reproducción directa del ideario panhispánico, nos interesa sugerir que, al menos, la forma en la que queda representado el español es llamativamente similar.

Finalmente, no queremos dar por concluido el análisis sin estudiar las características especificas con la que queda plasmado este "español general" en el texto primero. Especificamente, a los rasgos generales ya relevados en los párrafos anteriores, nos gustaría añadir algunos ejemplos que muestran zonas en las que esta variedad "artificial" se presenta esencialmente inestable. Consideremos, por ejemplo, los siguientes enunciados proferidos por Planc:

(5) Planc la Alfonsa, Antoni y al Padre Francisco]: Los franceses son muy licenciosos. Disculpad. (p. 39)

\section{(6) Planc [a Sanchis y Dimitril: Disculpen.} (p. 55)

(7) Planc (Ve salir al cavernícola y se dirige a Riera): Perdonad. Estoy teniendo problemas para administrar mi tiempo. Adelante, Cabo. (p. 16)

(8) Planc [A Aribau] (Se relaja): Es cierto. Perdona. No lo digo bien. Asunto concluido. (p. 29)

Por un lado, en (5) y (6) el mismo personaje en un contexto sintáctico idéntico (verbo en imperativo en oración independiente) utiliza dos formas diferentes de segunda persona del plural: en primer caso, la variante en "vosotros", típicamente peninsular; en el segundo, la que conjuga en "ustedes", empleada en variedades americanas. Por otro lado, en (7) y (8), enunciados atribuidos también a Planc, se apuesta a dos formas diferentes para marcar la segunda persona del singular en modo imperativo: la forma plural en "vosotros" "perdonad" en (7) y la conjugación del verbo en singular y en "tú" en (8). Nótese, además de la variación en los cuatro ejemplos, el uso de una variante plural en un contexto en el que la expresión remite a un único interlocutor.

Más aún, la obra presenta desplazamientos en otro orden de fenómenos. Obsérvese, por ejemplo, las siguientes réplicas a cargo de Aribau:

(9) Aribau: Pues dicen que le prendieron fuego [al hospital] con los enfermos adentro. (p. 20)

(10) Aribau: No puede ser. Yo mismo lo vi [a Planc] ponerla [lista] allí. (p. 126)

En (9) y (10) los enunciados, atribuidos al mismo personaje, presentan procedimientos lingüísticos distintos a propósito del uso de los pronombres átonos de tercera persona en función objeto directo. En el primer ejemplo, se reemplaza la palabra "hospital" por el pronombre "le". Este fenómeno, denominado leísmo, "abarca el área central y noroccidental de Castilla- junto con focos aislados en ciertos países hispanoamericanos" (DPD). En (8), en cambio, se emplea el pronombre "lo" para referirse al nombre propio "Planc" que cumple la función sintáctica objeto directo. Al respecto, nótese que, si bien en ambas muestras los pronombres reemplazan distintos tipos de sustantivos (un nombre común y un nombre propio, respectivamente), en ambos casos se trata de sintagmas nominales conjugados en masculino singular. En ese sentido, sería esperable que el pronombre átono que reemplace la expresión sea el mismo. Asimismo, en (5-10) los fenómenos en cuestión son del orden morfosintáctico y, en tanto pertenecientes a ese nivel gramatical, es llamativamente extraño que presenten alternancia en un mismo hablante.

Dejando de lado las particularidades gramaticales de los ejemplos, lo que nos interesa señalar es que los dos casos presentados generan una representación de este "español general" en 
la pieza como un sistema con zonas de gran inestabilidad. Esto, si bien puede explicarse por su carácter de lengua artificial, muestra la homologación de la obra con el discurso panhispánico. Como reseñamos, esta tradición tiende a enfatizar que las diferentes variedades del español son comprensibles entre si y, así, relega las particularidades de cada sistema a un segundo plano. En el caso de La terquedad, la libre alternancia de rasgos no se presenta como un hecho problemático y esto, por su parte, refuerza la inscripción de la obra en la tradición discursiva del panhispanismo.

\section{Conclusiones}

En este trabajo nos propusimos analizar qué representaciones emergen de las lenguas que se utilizan en La terquedad, texto dramático de Rafael Spregelburd. Como mencionamos, creemos que identificar estos valores asociados a las variedades es fundamental al momento de dar cuenta de las particularidades con las que emerge la figura del dramaturgo, ya que, por definición, él queda identificado con ellos. Para cumplir con nuestro objetivo, adoptamos una perspectiva polifónica y dialógica de la enunciación, la cual nos condujo a tomar distancia de la definición tradicional del concepto de representación, entendido por la sociolingüística y el análisis del discurso como una imagen de carácter mental que el hablante posee sobre algún objeto o situación. Por el contrario, en este artículo la adaptación de un enfoque argumentativo nos llevó a eliminar de la noción de representación cualquier elemento cognitivo e intencionalista y caracterizar esta unidad como una idea (o un conjunto de ideas) que sobre alguna entidad da el enunciado.

En cuanto al análisis del corpus, mostramos, en primer lugar, que la ausencia de notas al pie de página escenifica una imagen del inglés como una lengua conocida por el lector modelo. Esto, por su parte, nos permitió ligar esta representación al discurso social que usualmente circula sobre esta variedad, en el que es mostrada con un estatus de lengua internacional. En segundo lugar, observamos que la forma particular en que se organiza la alternancia español/valenciano permite identificar que esta última lengua emerge caracterizada como un sistema poco prestigioso que ocupa la posición subalterna en la situación diglósica que evoca el texto dramático. En tercer lugar, nos dedicamos a estudiar qué representación surge del español. Al respecto, observamos que la obra utiliza tres variedades distintas de esta lengua: un español peninsular en las notas al pie de página, un español americano en las didascalias y un "español general" en el texto primero. Asimismo, intentamos sugerir que el hecho de que el lector modelo quede mostrado como alguien que maneja las tres variedades, que estas convivan sin dificultad en la obra y la propia construcción de un "español general" permiten leer que la obra se homologa con los argumentos principales del discurso panhispánico. Sin embargo, un análisis detallado de las réplicas evidenció la presencia de zonas de inestabilidad en la creación de este lenguaje artificial, especificamente, la convivencia de rasgos de distintas variedades. Al respecto, intentamos poner en relación este hecho con la propia reproducción del modelo panhispanista que, al poner el acento en aquello que las variedades tienen en común, debilita la percepción de las diferencias entre ellas como un asunto a atender.

Quedará pendiente para futuros trabajos poner en relación los hallazgos aquí presentados con el análisis de las representaciones lingüísticas en otras obras de Spregelburd a los efectos de cotejar si existen similitudes o diferencias en los modos en que las lenguas quedan mostradas.

\section{Bibliografia}

ANSCOMBRE, Jean-Claude; DUCROT, Oswald. L'argumentation dans la langue. Bruselas: Mardaga, 1983

ARNOUX, Elvira. 'La lengua es la patria', 'nuestra lengua es mestiza' y 'el español es americano': desplazamientos significativos en el III Congreso de la Lengua Española. In: HOFMANN, Sabine (ed.). Medios, espacios y nuevas comunidades imaginadas. Berlin: Edition Tranvia, 2006, p. 17-39.

ARNOUX, Elvira. Representaciones sociolingüisticas y construcción de identidades colectivas en el Mercosur. In: CELADA, María Teresa; FANJUL, Adrián; NOTHSTEIN, Susana (Coords.). Lenguas en un espacio de integración. Acontecimientos, acciones, representaciones. Buenos Aires: Biblos, 2010, p. 17-38. 
ASOCIACIÓN DE ACADEMIAS DE LA LENGUA ESPAÑOLA Y REAL ACADEMIA ESPAÑOLA. La nueva política lingüistica panhispánica. Presentación en el III Congreso Internacional de la Lengua Española (CILE), 2004

AUTHIER, Jacqueline. Hétérogénéité(s) énonciative(s). Languajes, v. 73, p. 98-111, 1984. https://doi.org/10.3406/ lgge.1984.1167

BAJTÍN, Mijail. Estética de la creación verbal. México: Siglo XXI, 1985

BEIN, Roberto. Las lenguas como fetiche. In: PANESI, Jorge; SANTOS, Susana (ed.). Actas del Congreso internacional Debates actuales: las teorías criticas de la literatura y la lingüistica. Buenos Aires: Facultad de Filosofia y Letras, Universidad de Buenos Aires, 2005, s/p.

CAREL, Marion; DUCROT, Oswald. La semántica argumentativa: una introducción a la teoria de los bloques semánticos. Buenos Aires: Colihue, 2005.

DUCROT, Oswald. El decir y lo dicho. Buenos Aires: Paidós, 1984

ECO, Umberto. Lector in fabula. Barcelona: Lumen, 1981.

FASOLD, Ralph. La sociolingüistica de la sociedad. Madrid: Visor Libros, 1996.

FISHMAN, Joshua. Sociolinguistics: a brief introduction. Rowley: Mass, Newbury House, 1970.

FERGUSON, Charles. Diglossia. Word, v.15, n. 2, p. 325-340, 1959. https://doi.org/10.1080/00437956.1959.11659702

GALLARDO, Andrés. Hacia una teoría del idioma estándar. R.L.A. Revista de lingüística aplicada, v. 16, p. 85-120, 1978.

GARCIA NEGRONI, Maria Marta. Dialogismo y polifonía enunciativa. Apuntes para una reelaboración de la distinción discurso / historia. Páginas de Guarda. Revista de Lenguaje, Edición y Cultura Escrita, v. 7. p. 15-31, 2009.

GARCIA NEGRONI, Maria Marta. Polifonía, evidencialidad y descalificación del discurso ajeno. Acerca del significado evidencial de la negación metadiscursiva y de los marcadores de descalificación. Letras de Hoje, v. 51, n. 1, p. 7-16, 2016a. https://doi.org/10.15448/1984-7726.2016.1.23851.

GARCIA NEGRONI, Maria Marta. Discurso politico, contradestinación indirecta y puntos de vista evidenciales. La multidestinación en el discurso político revisitada. Revista ALED, v. 16, n. 1, p. 37-59, 2016b. https://doi. org/10.35956/v.16.n1.2016.p.37-59.

GARCIANEGRONI, Maria Marta. Argumentación y puntos de vista evidenciales citativos. Acerca de la negación metadiscursiva en el discurso político. Oralia, v. 21, p. 223-236, 2018.

GARCIA NEGRONI, María Marta. El enfoque dialógico de la argumentación y la polifonía, puntos de vista evidenciales y puntos de vista alusivos. Rilce. Revista de Filologia Hispánica, v. 35, n. 2, p. 521-549, 2019. https:// doi.org/10.15581/008.35.2.521-49.

GARCÍA NEGRONI, María Marta; LIBENSON, Manuel; MONTERO, Ana Soledad. De la intención del sujeto hablante a la representación polifónica de la enunciación. Acerca de los límites de la noción de intención en la descripción del sentido. Revista de Investigación Lingüistica, v. 16, p. 237-262, 2013.
LAPESA, Rafael. Historia de la lengua española. Madrid: Gredos, 1984.

LAURIA, Daniela; LÓPEZ GARCÍA, María. Instrumentos linguisticos académicos y norma estándar del español: la nueva política linguistica panhispánica. Lexis, v. XXXIII, n. 1, p. 49-89, 2009.

LAURIA, Daniela. Los nuevos instrumentos linguísticos de la Academia Argentina de Letras. Posibilidades de acción, alcances, limites y tensiones en relación con la norma panhispánica. Anuario de Letras. Linguistica y Filologia, v. VI, p. 155-186, 2018. https://doi.org/10.19130/ iifl.adel.6.1.2018.1480.

LOPEZ GARCIA, Maria. Nosotros, vosotros, ellos. La variedad rioplatense en los manuales escolares. Buenos Aires: Miño y Dávila editores, 2015.

MOSCOVICl, Serge. El Psicoanálisis, su imagen y su público. Buenos Aires: Editorial Huemul, 1979.

PÊCHEUX, Michel. Las verdades evidentes: lingüistica, semántica, filosofía. Buenos Aires: Ediciones del CCC Centro Cultural de la Cooperación Floreal Gorini, 2016.

RAITER, Alejandro. Representaciones sociales. In: RAITER, Alejandro. Representaciones sociales. Buenos Aires: Eudeba, 2001, p. 11-29.

REAL ACADEMIA ESPAÑOLA Y ASOCIACIÓN DE ACADEMIAS DE LA LENGUA ESPAÑOLA. Diccionario Panhispánico de Dudas. Bogotá: Santillana, 2005.

SPREGELBURD, Rafael. La terquedad. Buenos Aires: Atuel, 2009.

SZURMUK, Monica y IRWIN, Robert McKee (Coord.). Diccionario de estudios culturales latinoamericanos. Buenos Aires: Siglo XXI Editores e Instituto Mora, 2009.

WOOLARD, Kathryn. La autoridad lingüística del español y las ideologias de la autenticidad y el anonimato. In: DEL VALLE, José (ed.). La lengua, ¿patria común? Ideas e ideologias del español. Madrid/Frankfurt: Iberoamericana/Vervuert, 2007, p. 129-142.

ZUCCHI, Mariano. Subjetividad autoral en El pánico de Rafael Spregelburd. In: LÓPEZ, Liliana; ZUCCHI, Mariano (ed.). Topologias de la crítica teatral V. CABA: Departamento de Artes Dramáticas (UNA), 2018, p. 79-94. https://doi.org/10.31819/9783865278999-008.

ZUCCHI, Mariano. Construcción del ethos autoral en La terquedad de Rafael Spregelburd. Análisis de la figura del Locutor-Dramaturgo y su posicionamiento enunciativo. Reflexión Académica en Diseño y Comunicación, v. XXXIX, p. 96-101, 2019a.

ZUCCHI, Mariano. Subjetividad autoral en La estupidez de Rafael Spregelburd. La puesta en escena de un ethos intelectual como un mecanismo de legitimación enunciativa. In: MARI, Clara; ZUCCHI, Mariano (ed.). Dispositivos para el análisis del texto dramático y espectacular. Topología de la critica teatral VI (Directora Liliana López). Buenos Aires: Departamento de Artes Dramáticas, Universidad Nacional de las Artes, 2019b, p 141-156. https://doi.org/10.15446/fyf.v33n2.88470.

ZUCCHI, Mariano. Una clasificación del discurso didascálico desde una perspectiva polifónica de la enunciación. Revista Letrónica, v. 11, n. 4. p. 452-464, 2019 c. https://doi.org/10.15448/1984-4301.2018.4.31274. 


\section{Mariano Nicolás Zucchí}

Es Licenciado en Letras (Universidad de Buenos Aires). Actualmente se encuentra terminando sus estudios de doctorado en la Universidad Nacional de las Artes (Beca CONICET). Se desempeña como Jefe de trabajos prácticos en las materias Análisis del Texto Teatral I e Historia del Teatro Moderno y Contemporáneo (UNA).

\section{Dirección}

Mariano Nicolás Zucchi

Universidad de Buenos Aires

Viamonte 430, C1053

Ciudad Autónoma de Buenos Aires, Argentina. 